\title{
Design of forms for clinical trials (1)
}

\author{
PATRICIA WRIGHT, JOHN HAYBITTLE
}

British Medical fournal, 1979, 2, 529-530

Clinical trials rely heavily on forms to collect data for statistical analysis. Nevertheless, accurate completion of such forms depends on how carefully the form has been designed. We have studied research findings which show how design factors influence form-fillers, and we suggest procedures for improving form design.

\section{Introduction}

The success of most clinical trials depends on accurate completion of forms. Verification procedures may detect omissions and inconsistencies, but many recorded data are critically dependent on the accuracy of the form-filler. Recent reviews of behavioural research studies are relevant to the comprehension of written materials in general, ${ }^{2}$ and the design of forms in particular. ${ }^{3}$ This latter review showed that, even though the form-filler understood the question and knew the answer, the information entered on the form was not necessarily correct. The implication of this for clinical trials is that careful design of forms may be almost as necessary as having adequate randomisation procedures.

The specific problems of designing forms for clinical trials fall into three categories. These relate to: (1) the content of the form, and whether certain questions should be asked at all;

\section{MRC Applied Psychology Unit, Cambridge CB2 2EF}

PATRICIA WRIGHT, PHD, scientist

\footnotetext{
Department of Physics, Addenbrooke's Hospital, Cambridge CB2 2QQ

JOHN HAYBITTLE, PHD, chief physicist
}

(2) the completion of the form, including the layout of the page and the methods of answering questions; and (3) the coding of the completed answers for subsequent data analysis. In addition to these three categories there are issues about the procedures required for constructing a trial form. Design guidelines may be based on available research findings. Such guidelines, however, cannot guarantee that a particular form will be adequate: other kinds of evaluation are necessary.

\section{Deciding about the content}

The temptation is to collect more clinical data than are absolutely necessary. Such temptation should be resisted. To quote Richard Peto," "The statistician should, at the design stage, cross out from the draft coding forms most of the things that the trial organiser thinks he wants to ask." The reasons are twofold. Firstly, the amount of associated paperwork is likely to influence the willingness of a consultant to enter a patient in the trial. Secondly, the quality of the response may deteriorate if too much is demanded of a busy clinician.

One partial solution to this problem is to differentiate on the form the information that only the clinician can provide from the information that could be provided by other staff such as a nurse or clerk, or by a computer. One example of the latter's use is the supply of computer-printed, self-adhesive labels for sticking on the tops of follow-up forms, thus eliminating the need for repeatedly entering the same information about the patient. "When developing forms for use in clinical trials it is certainly necessary to consider carefully who will be completing them and in what circumstances. A form completed in the patient's presence may call for much briefer responses than information filled in subsequently. It may be possible to arrange the questions on the form to correspond with the logical or temporal sequence in which the information becomes available to the form-filler.

Issues about content depend not only on the hypothesis being 
tested and the design of the trial but also on providing reliable administrative procedures. Accurate identification of the patient may be helped by including the National. Health Service number on the first page. If there is a risk that the pages will become separated it may also be advisable to identify the patient by name or number on each page of the document.

Issues about content persist, even when it has been decided that a particular kind of information is needed for the analysis. The information wanted and the information asked for on the form do not have to be exactly the same. A good example of this is age. It is preferable to record the patient's date of birth (as this is necessary for follow-up using national archives) rather than age, which can easily be worked out on the computer when required. Similarly, it is an issue of content to specify how precise the answers should be. Sometimes it is left to the discretion of the form-filler whether he rounds to whole numbers or uses decimal places when recording height, weight, and surface area. Not only does this raise doubts in the formfiller's mind: it also increases the difficulty of the data processor's task. As well as punching the information someone must be given the responsibility for taking decisions about it.

Dates also raise questions about the exactness of the information required. Date of assessment and of onset of symptoms can hardly be given with the same precision. On the other hand, precision is of cen lost unnecessarily-as, for example, when a question asks the form-filler to grade into three or four adjectives of degree those measurements which can be expressed numerically. The use of category scales such as "Improved, unchanged, deteriorated" lcaves recm for considerable variation among clinicians. Patient's suljective judgments are sometimes recorded by using continuous analogue scales." ${ }^{6}$ The problems of constructing and interpreting the data from such scales have been discussed elsewhore. '

Once ciccisions have been taken about what information should be collected, the next problem concerns the presentation of the material. It is this which largely determines how casily the form can be completed, and it is here that the findings of bchavioural rescarch may te applied.

The ease of completing a form depends partly on the language used and the typographic presentation of the form, and partly on constraints which limit the way answers are given to the qucstions.

\section{Linguistic factors}

On many forms used in clinical trials the language is not a major problem. In this respect, trial forms differ from the numcrous other forms that the public complete-fcr example, when applying for insurance or welfare benefits. Understanding clinical forms is helpcd by using conventional, professional terminolcgy. Nevertheless, when questions include comparative terms, some ways of asking the question are easier to answer than others-for excmple, research has shown that positive terms such as better, longer, bigger, more are all easier to understand than negative terms such as worse, shorter, smaller, less. ${ }^{.10}$ The differential case cf these antonyms may result in a $20^{\prime \prime}$ " difference in the accuracy of answering questions.

It has also been found that the gap between positive and negative terms widencd as the difficulty of the other elements in the task increased. Similar potentiation might be expected when people are tired or when the environment is distracting. Whonever possible, it secms preferable, therefore, to phrase yes/no questions with their focus on the easier comparative terms whenever possible.

One other linguistic factor that may be a source of difficulty on clinical trial forms is reliance on small function words, such as prepositions, to convey critical aspects of the meaning. Th. table illustrates how, in the context of a negative verb such as reduce and a negative comparative less, the form-filler must pay close attention to the word by. The clinician in a hurry, who knows that the present tumour size is now less than half its original size, may inadvertently circle option (3) ("Reduction of tumour by less than $50^{\prime \prime}$ ",), misinterpreting the by as to while coping with the negatives. Perhaps a safer phrasing for alternatives (3) and (4) would be:

(3) Smaller, but not as small as half the original diameter.

(4) Smaller, now less than half the original diameter.

Problems when negatizes are used

Date of next attendance

Day Month Year

General condition

(1) At work or normal domestic activity

(2) Full activity but not at work
(3) Out and about, but activity restricted

(4) Confined to home or hospital

(5) Bed-ridden

(6) Dead

Please circle

Tumour mass

(1) Larger or new lesions (if so, patient may be taken off treatment regimen, (2) Unchanged

Reduction of tumour diameter by less than $50^{\circ}$

4) Reduction by more than 50 "

Please circle

An alternative solution would be to ask the clinician to record the physical size of the tumour. Computer software could then generate the required categories. It has already been pointed out that there are usually advantages in recording direct numerical measurements rather than asking the form-filler to make broad category judgments.

The importance of the layout of a form, together with the effects of requiring the form-filler to answer in different ways, will be considered in the next article in this series.

We thank Dr fohn Woodhiad-Galloway of the Medical Research Council for encouraging us to arite this series of articles.

\section{References}

${ }^{1}$ Hartley, J, Designing Instructional Text. London, Kogan Page, 1978.

2 Wright, P, in Psychology Law and Legal Processes, vol 2, ed S LloydBostock. London, Macmillan (in press).

3 Wright, P, in Visual Presentation of Information, a NATO Conference, Netherlands, 1978 (proceedings in press).

1 Peto, R, Biomedicine Special Issue, 1978, 28, 24.

Fayers, P M, paper presented at MRC Cancer Trial Strategy Meeting, Oxford, 1978.

"Bond, A, and Lader, M H, British Fournal of Medical Psychology, 1974, 47, 211 .

' Brigham, F R, Applicd Ergonomics, 1975, 6, 90.

* Oborne, D J, and Clarke, M J, Applied Ergonomics, 1975, 6, 97.

${ }^{9}$ Clark, H H, Psychological Review, 1969, 76, 387.

I" Clark, H H, and Clark, E V, in Psychology and Language. New York, Harcourt Brace, Jovanovich, 1977.

Is the uptake of the ordinary BCG vaccine as good as INH-resistant $B C G$ when used in babies along with isoniazid treatment?

There is no clear evidence that the usual immunity would be induced by ordinary BCG when a baby is simultaneously receiving isoniazid treatment. It is extremely unlikely that there would be sufficient numbers for a controlled study which would permit comparison between the use of ordinary BCG and isoniazid-resistant BCG in these circumstances. The development of immunity does seem to require some persistence of $B C G$ in the tissues. ${ }^{1}$ We do know that isoniazid kills ordinary BCG, and it would certainly seem much wiser to use isoniazid-resistant BCG whenever isoniazid treatment is given at the same time.

Lefford, M J, American Review of Respiratory Disease, 1978, 117, 103. 